\title{
Fairness norm through social networks: a simulation approach
}

\author{
Omar Rifki ${ }^{*}$ (D) and Hirotaka Ono ${ }^{2}$
}

\section{*Correspondence:}

omar.rifki@gmail.com

${ }^{1}$ Departement 14S, Centre

CIS, Mines Saint-Etienne,

42023 Saint-Etienne, France

Full list of author information

is available at the end of the

article

\begin{abstract}
Recently there has been an increased interest in adopting game-theoretic models to social norms. Most of these approaches are generally lacking a structure linking the local level of the 'norm' interaction to its global 'social' nature. Although numerous studies examined local-interaction games, where the emphasis is placed on neighborhood relations, regarding social network as a whole unique entity seems to be quite limited. In this paper, we conduct a series of simulation experiments to examine the effects that a network topology could have on the speed of emergence of the social norm. The emphasis is placed on the fairness norm in the ultimatum game context, by considering three network type models (Barabási-Albert, Watts-Strogatz and Erdős-Rényi) and several intrinsic topological properties.
\end{abstract}

Keywords: Social norm, Ultimatum game, Social network, Simulations

\section{Introduction}

Social norms (SN) are customary rules of behaviors that coordinate people's interactions with each others, as defined by Young in [10]. They are different from mere conventions and fashions; they could in fact guide our collective behaviors in social situations, even at the expense of our individual interest [2]. Such self-enforcing normative power has been the target of several studies in philosophy, social psychology, political science and economics.

In the current paper, we focus on the game-theoretic approaches to $\mathrm{SN}$, given that an increasing number of related studies have been reported in recent years [22]. In this setting, a $\mathrm{SN}$ is primarily perceived as a Nash equilibrium of a symmetric game with multiple equilibria, usually a coordination game. One stream of thoughts based on evolutionary game theory considers a given $\mathrm{SN}$ to be the result of an evolutionary process of expectation formation that has the ability to solve the equilibrium selection problem for coordination games, or in loose terms a 'focal point' [23] shaped through evolution [6, 28]. On the other hand, Bicchieri [5] regards SNs as a class of default 'behavioral rules', that are triggered once and only once the individuals have collectively the right kind of expectations about norm conformity. Bicchieri talks about "conditional preferences for obeying the norms". Two expectations for conditional preferences are specified: empirical expectation, where the individual believes

(c) The Author(s), 2021. Open Access This article is licensed under a Creative Commons Attribution 4.0 International License, which permits use, sharing, adaptation, distribution and reproduction in any medium or format, as long as you give appropriate credit to the original author(s) and the source, provide a link to the Creative Commons licence, and indicate if changes were made. The images or other third party material in this article are included in the article's Creative Commons licence, unless indicated otherwise in a credit line to the material. If material is not included in the article's Creative Commons licence and your intended use is not permitted by statutory regulation or exceeds the permitted use, you will need to obtain permission directly from the copyright holder. To view a copy of this licence, visit http:// creativecommons.org/licenses/by/4.0/. 
that a sufficient subset of the population conforms to the norm and normative expectation, where the individual believes that a sufficient subset of the population expects him to conform to the norm. The latter expectation can be combined with possible sanctions. Once the norm conforming state is triggered, SNs are manifested as equilibria of coordination games in this case also. Other approaches to SN involve signaling systems [17]. There are major disagreements among these game-theoretic models. For instance, whether SNs should affect individual's utility or not. Such disagreements and the failure to tackle some key challenges have led Paternotte and Grose [22] to conclude that game theory may have a limited explanatory power to account for social norms.

On another front, social network analysis has been used with considerable success in order to model social ties within a population, a group of people [11, 18]. It allows to formalize using the graph theoretic language the structure of interactions and relationships (edges) between individuals (vertices), and to subsequently study phenomena in these structures, such as the six degrees of separation and the coexistence of weak / strong ties. In cases of strategic interactions, the situations where an individual plays a game with its neighbors by targeting to coordinate with as many as possible of them, are known as local-interaction games $[12,21]$. They have been extensively covered in the literature (see Weidenholzer's survey [27] and Goyal's book [18]). If an equilibrium happens in these game for a given population, it shall describe a social norm. However, these approaches account above all for the local level of graphs, the relations linking neighbors. Considering the social network structure as a whole and unique entity, other than in a global context where any player can be linked to any other player, or other than regular graphs such as circles, grids and lattices, is quite limited in these accounts. As known, the network structure might vary from a population to another one, and might be much different from regular graphs. Besides, the interactions within a population are in a dynamic state, that could transform the network shape in times [24]. For these reasons, it is essential to perceive social network in a large-scale outlook when dealing with social norms.

A key social norm in human societies is fairness. It plays a central role in the allocation of various goods, the promotion of social order, and the group cohesion. There is a substantial evidence suggesting that the fairness norm is common within societies, e.g., in the labor market [4]. However, its interpretation is rather context-dependent, and may suggest different behaviors depending on the situation at hand, and also on the cultural shared scripts. The game of choice to study fairness is the ultimatum bargaining game and its derivations $[14,15]$. One advantage of such a game is that the contextual information clues are kept at their minimal level, and fairness in this setting is usually understood by all players. In the current study, in order to study the fairness norm, which is central to human coordination, we focus on the ultimatum game, but in a more realistic outlook accounting for the social network connection.

Concerning the SN model, we opt for Bicchieri's approach, considering that several experimental results have been already obtained in her case. We conduct a series of simulation experiments to examine the effects that a network topology could have on the speed of emergence of social norms. The emphasis is placed on three realistic models of social networks and a number of intrinsic topological properties in the emergence of the fairness norm, which will be seen in the next section. 


\section{Methodology}

The ultimatum game is a typical bargaining game with two players: the proposer $(\mathrm{P})$ and the responder (R). The purpose of the game is to split an amount of money $M$ between $\mathrm{P}$ and $\mathrm{R}$, where $\mathrm{P}$ first proposes a division $0 \leq x \leq M$ of money to $\mathrm{R}$. The latter player can either accept or refuse the offer. In case the offer $x$ is accepted, $\mathrm{P}$ gets $(M-x)$ and $\mathrm{R}$ gets $x$, otherwise both players will receive nothing. What is interesting about this game is that laboratory experiments of its one-shot version, which is the one considered in this manuscript, contradicts the standard economic theory and its well-known principle of expected utility maximization. On average around $M / 3$ to $M / 4$ is offered, with a sizable proportion of people offering $M / 2$ [19]. When the fair division $M / 2$ is adopted by the overall population, this strategy shall be interpreted as the fairness norm. To study the evolution dynamics leading to the fairness SN or to an unfair norm, we use the approach introduced by Bicchieri in her book [5], which is presented in section "Norm emergence". Section "Proposed relaxations" discusses the relaxations of the approach concerning the common shared information between players, and the structure of the social network.

\section{Norm emergence}

In Bicchieri's model, norms are internalized, thus their effect is directly accounted in players' utilities. If the player $i$ is assigned the role $\mathrm{P}$, its utility for a strategy $x \in S$, where $S \subseteq[0, M]$ is the set of considered strategies, is written as follows:

$$
U_{P, \text { accept }}^{i}(x)=(M-x)+ \begin{cases}0 & \text { if } x \geq N_{i} \\ -k_{i}^{\prime}\left(N_{i}-x\right) & \text { otherwise }\end{cases}
$$

while the utility of the player $i$ in the role $R$ is equal to,

$$
U_{R, a c c e p t}^{i}(x)=x+ \begin{cases}0 & \text { if } x \geq N_{i} \\ -k_{i}\left(N_{i}-x\right) & \text { otherwise }\end{cases}
$$

$N_{i}$ is the estimate of the social norm within the population of players $\mathbb{P}$, as perceived by player $i$. The parameters $k_{i}^{\prime}$ and $k_{i}$ are the sensitivities to the norm when player $i$ is, respectively, in the role of the proposer and the responder. The responder sensitivity $k_{i}$ lies within the range $\left[0, k_{\max }\right]$. It induces a disutility proportional to the deviation from the perceived norm $\left(N_{i}-x\right)$, when $x<N_{i}$. In case $k_{i}$ is large, the player $i$ has a strong inclination to conform to the $\mathrm{SN}$, and tends then to reject low offers. In fact, the values of $k_{i}$ and $N_{i}$ define a lower bound for accepted offers by player $i$, $U_{R, a c c e p t}^{i}(x) \geq 0 \Longrightarrow x \geq \frac{k_{i}}{1+k_{i}} N_{i}$. The value of $k_{\max }$ delimits the variations of $k_{i}$, and is chosen to be in the same range of $M^{1}$. The parameter $k_{i}$ is chosen from a uniform distribution on $\left[0, k_{\max }\right]$. The second parameter, the proposer sensitivity $k_{i}^{\prime}$, is selected from a normal distribution with a mean equal to 0 and a standard deviation of 0.2 , in accordance with [5]. In the same manner of $k_{i}$, the parameter $k_{i}^{\prime}$ controls also the degree of conformity to the perceived norm. A high $k_{i}^{\prime}$ implies that $i$ is more inclined

\footnotetext{
${ }^{1} k_{\max }$ can take larger values than $M$. It corresponds to the situation where the responder $i$ is highly sensitive to the perceived norm $N_{i}$, and can have a null utility for offers deviating very slightly from the perceived norm $x=N_{i}-\epsilon$ : $U_{P, \text { accept }}^{i}\left(N_{i}-\epsilon\right)=0 \Longrightarrow k_{\max }=\frac{N_{i}}{\epsilon}-1 \leq \frac{M}{\epsilon}-1$, for $\epsilon>0$ small enough.
} 
to conform to the $\mathrm{SN}$. In order to not associate a positive utility to deviation from $N_{i}$, which means that the proposer benefits from deviating from the $\mathrm{SN}$, only positive values of $k_{i}^{\prime}$ are considered (as in [5]). Note that in the rejection case, the utility terms are $U_{P, \text { reject }}^{i}(x)=U_{R, \text { reject }}^{i}(x)=0$.

The distributions of $k_{i}$ and $k_{i}^{\prime}$ are not symmetric, since they are related to the same term, i.e., the deviation $\left(N_{i}-x\right)$ from the point of view of the responder, but from different perspectives. When player $i$ is a responder, the sensitivity $k_{i}$ can be seen as his type. To represent population's types, sampling from a uniform distribution is a reasonable choice. However, when $i$ is in the proposer role, the sensitivity $k_{i}^{\prime}$ is seen as a faculty of $i$ to account for the opponent interest during the interaction. Such faculties are often distributed according to the normal distribution, e.g., the empathy quotient [20].

The steps of Bicchieri's approach are listed in Algorithm 1. In the following, we describe how a player $i$ estimates the norm $N_{i}$ at each iteration of Algorithm 1, which corresponds to the step 2 of the algorithm. The set of strategies in the population is set to $S=\{0,1, \ldots, M\}$ (as in [5]). At each interaction, the distribution $P$ of the offers $x$ in the whole population for the previous interaction is considered to be accessible and known to all players. Each individual has been assigned a threshold $0 \leq t_{i} \leq 1$, chosen from a continuous uniform distribution on [0,1], which represents for $i$ the minimal proportion of the population that must adopt the same strategy before this latter to be considered a candidate for the social norm. The first step in $N_{i}$ computation is to compute the set of strategies to be considered for the social norm,

$$
n_{i}=\left\{x \in S: P(x)>t_{i}\right\} .
$$

Then, a new distribution of offers representing the player $i$ own beliefs for the current norm in the society is constructed. To do so, the distribution $P$ is restricted to the set $n_{i}$ and trimmed according to the threshold $t_{i}$ as follows:

$$
P_{n_{i}}(x)= \begin{cases}\kappa\left(P(x)-t_{i}\right) & \text { if } x \in n_{i} \\ 0 & \text { otherwise }\end{cases}
$$

where $\kappa$ is a renormalization constant for the distribution $P_{n_{i}}$. If $n_{i}=\emptyset$, no candidate for the $\mathrm{SN}$ can be derived, thus all strategies can be considered in this case. Finally, the constructed probability distribution by the player $i$ can be written as:

$$
P_{i}= \begin{cases}P_{n_{i}} & \text { if } n_{i} \neq \emptyset \\ P & \text { otherwise }\end{cases}
$$

and the player's estimation of the norm $N_{i}$ is equal to the expected value of the distribution $P_{i}$,

$$
N_{i}=\sum_{j=0}^{M} j \times P_{i}(j) .
$$

To illustrate the computation of $N_{i}$, let us consider an example run of Algorithm 1 with $N=5, M=10, k_{\max }=10$, and $I=2$. Table 1 shows the initial values which are randomly generated. The updates of the distribution of strategies $P$ for the three iterations of the algorithm are listed in Table 2, in addition to showing the constructed 
Table 1 Input values of an instance run of Algorithm 1 with $N=5, M=5$, and $k_{\max }=10$

\begin{tabular}{lllll}
\hline Player $i$ & Threshold $\boldsymbol{t}_{\boldsymbol{i}}$ & Responder sensitivity $\boldsymbol{k}_{\boldsymbol{i}}$ & Proposer sensitivity $\boldsymbol{k}_{\boldsymbol{i}}^{\prime}$ & $\begin{array}{l}\text { Initial } \\
\text { strategy } \\
\boldsymbol{s}_{\boldsymbol{i}}\end{array}$ \\
\hline 1 & & & & 4 \\
2 & 0.565 & 1.00 & 0.002 & 9 \\
3 & 0.173 & 5.51 & 0.013 & 7 \\
4 & 0.479 & 3.01 & 0.019 & 7 \\
5 & 0.022 & 5.63 & 0.007 & 3 \\
\hline
\end{tabular}

Table 2 The update of the probability distributions $P_{1} P_{1}$, and $P_{4}$, plus the norm estimations $N_{1}$ and $N_{4}$

\begin{tabular}{|c|c|c|c|c|c|c|c|c|c|c|c|c|c|}
\hline Iteration & Distribution & $x=0$ & 1 & 2 & 3 & 4 & 5 & 6 & 7 & 8 & 9 & 10 & $N_{i}$ \\
\hline \multirow[t]{3}{*}{1} & $P$ & 0 & 0 & 0 & 0.2 & 0.2 & 0 & 0 & 0.4 & 0 & 0.2 & 0 & - \\
\hline & $P_{1}$ & 0 & 0 & 0 & 0.2 & 0.2 & 0 & 0 & 0.4 & 0 & 0.2 & 0 & 6 \\
\hline & $P_{4}$ & 0 & 0 & 0 & 0.195 & 0.195 & 0 & 0 & 0.414 & 0 & 0.195 & 0 & 6.02 \\
\hline \multirow[t]{3}{*}{2} & P & 0 & 0 & 0 & 0 & 0 & 0.8 & 0.2 & 0 & 0 & 0 & 0 & - \\
\hline & $P_{1}$ & 0 & 0 & 0 & 0 & 0 & 1 & 0 & 0 & 0 & 0 & 0 & 5 \\
\hline & $P_{4}$ & 0 & 0 & 0 & 0 & 0 & 0.814 & 0.186 & 0 & 0 & 0 & 0 & 5.18 \\
\hline \multirow[t]{3}{*}{3} & P & 0 & 0 & 0 & 0 & 0.6 & 0.4 & 0 & 0 & 0 & 0 & 0 & - \\
\hline & $P_{1}$ & 0 & 0 & 0 & 0 & 1 & 0 & 0 & 0 & 0 & 0 & 0 & 4 \\
\hline & $P_{4}$ & 0 & 0 & 0 & 0 & 0.604 & 0.395 & 0 & 0 & 0 & 0 & 0 & 4.37 \\
\hline Output & $P$ & 0 & 0 & 0 & 0 & 1 & 0 & 0 & 0 & 0 & 0 & 0 & - \\
\hline
\end{tabular}

distributions by players 1 and 4: $P_{1}$ and $P_{4}$. Player 1 has a threshold equal to $t_{1}=0.565$, which is larger than all the initial values of $P(x)$, then $n_{1}=\emptyset$, and subsequently $P_{1}=P$ and $N_{1}=E(P)=6$. In the second iteration, only $P(x=5)$ is larger than $t_{1}$, which leads for the constructed distribution to be $P_{1}(x=5)=1$ and $P_{1}(x \neq 5)=0$, and therefore $N_{1}=5$. In the same way, we obtain $N_{1}=4$ for the third distribution, . The player 4 has a low threshold $t_{4}=0.022$, which leads the set $n_{4}$ to include all strategies $x$ with $P(x) \neq 0$ for all iterations. Then, the updates of $P_{4}$ follows the formula (1), before computing $N_{4}$ as the expected value of $P_{4}$. The final output distribution $P$ is shown in the last row of Table 2. The social norm for this case is equal to 4 .

Table 3 The number $Q$ of players who accepted the offers of players 1 and 4 in step 3 of Algorithm 1, and the corresponding games

\begin{tabular}{llll}
\hline Iteration & Player & Q & Instance games \\
\hline 1 & 1 & 0 & - \\
& 4 & 2 & $4(P) \rightarrow 3(\mathrm{R}) ; 4(\mathrm{P}) \rightarrow 1(\mathrm{R})$ \\
2 & 1 & 2 & $1(\mathrm{P}) \rightarrow 2(\mathrm{R}) ; 1(\mathrm{P}) \rightarrow 2(\mathrm{R}) ;$ \\
3 & 4 & 3 & $4(\mathrm{P}) \rightarrow 2(\mathrm{R}) ; 4(\mathrm{P}) \rightarrow 5(\mathrm{R}) ; 4(\mathrm{P}) \rightarrow 2(\mathrm{R})$ \\
& 1 & 0 & - \\
& 4 & 3 & $4(\mathrm{P}) \rightarrow 2(\mathrm{R}) ; 4(\mathrm{P}) \rightarrow 1(\mathrm{R}) ; 4(\mathrm{P}) \rightarrow 1(\mathrm{R})$ \\
\hline
\end{tabular}




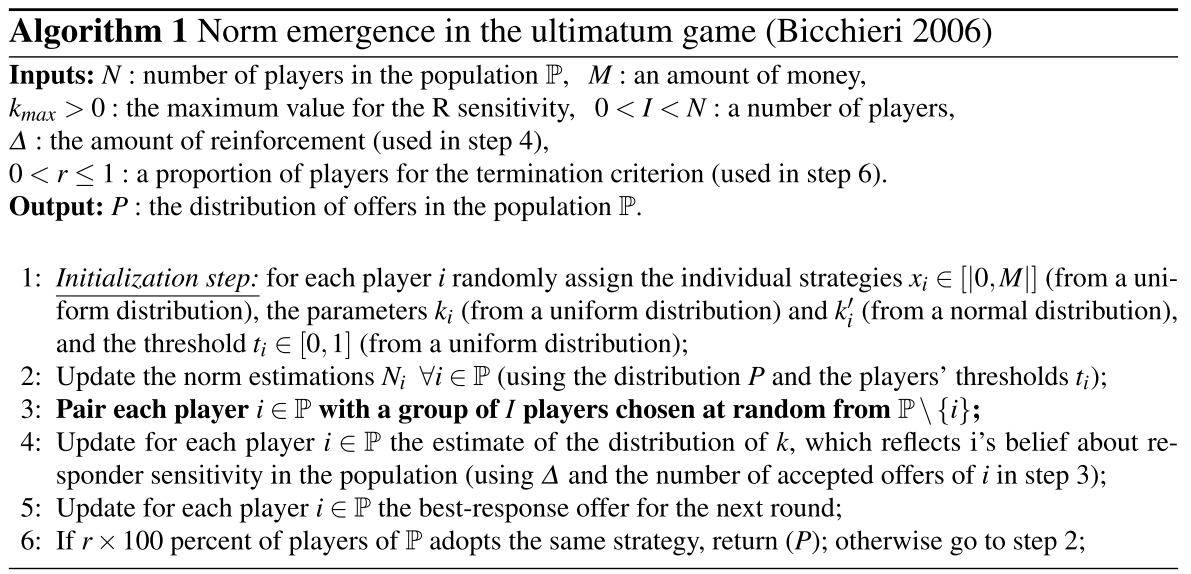

In the third step of Algorithm 1, each individual $i$ is paired with $I \in[|1, N-1|]$ players selected uniformly at random without replacement from the population $\mathbb{P} \backslash\{i\}$. The roles of the proposer and the responder are assigned at random as well for each pairwise interaction between $i$ and $j \in I$. Without loss of generality, let us suppose that $\mathrm{P}$ is player $i$ and $\mathrm{R}$ is player $j$. The proposer $i$ offers an amount $x_{i}$ to the responder $j$, who accepts it in case $U_{R, \text { accept }}^{j}\left(x_{i}\right)>U_{R, \text { reject }}^{j}\left(x_{i}\right)$ or in other terms:

$$
k_{j}<\frac{x_{i}}{N_{j}-x_{i}} \text {. }
$$

The division $x_{i}$ that each player $i \in \mathbb{P}$ proposes is updated in the step 5 of Algorithm 1 . The stochasticity of the algorithm in the initialization step (step 1) and in the paring step (step 3) renders the players learning process stochastic as well and nondeterministic.

In the fourth step, each player $i \in \mathbb{P}$ infers a distribution of the responder sensitivity $k$ in the population, based on its interactions in the previous step. The distribution of $k$ is used in the step 5 to derive the player $i$ 's best-response offer. In the current iteration, when $i$ plays the role of the proposer and its offer $x_{i}$ is accepted, he could infer an upper bound of the value of $k$ in $\mathbb{P}$ according to the inequality (2). In the absence of the subjective information $N_{j}$, the player $i$ could project his own view of the $\mathrm{SN}^{2}$. Then, i's belief about the responder parameter $k$ is falling within the range $\left[0, \frac{x_{i}}{N_{i}-x_{i}}\right.$. The new update of player i's distribution function of $k$ based on the current distribution function $f_{i}(k)$ is given by the normalization: $f_{i}^{\prime \prime}(k)=f_{i}^{\prime}(k) / \int_{0}^{k_{\max }} f_{i}^{\prime}(k) d k$, of the following function:

$$
f_{i}^{\prime}(k)=f_{i}(k)+ \begin{cases}Q \Delta \text { if } k<\frac{x_{i}}{N_{i}-x_{i}} \\ 0 \quad \text { otherwise }\end{cases}
$$

with $\Delta$ and $Q$ denoting, respectively, the amount of reinforcement, and the number of individuals who accepted player $i$ 's offer in the current iteration. This updating in nonBayesian since the actual responder value $k$ is not known. Table 3 shows the values of $Q$

\footnotetext{
2 There is evidence in the psychological literature that people project their own beliefs and preferences in the absence of information about other people's beliefs and preferences [5].
} 


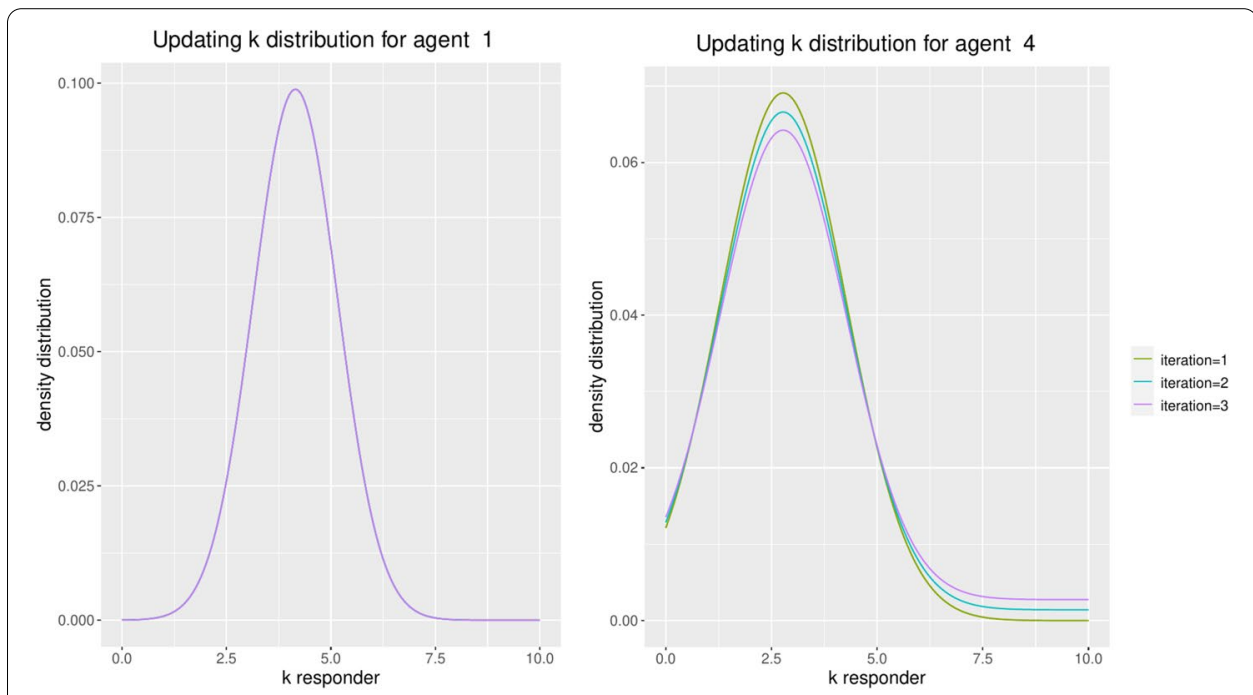

Fig. 1 The updates of the belief distribution $f(k)$ for players 1 and 4 using $\Delta=5 \times 10^{-3}$

for players 1 and 4 along the iterations of Algorithm 1, and their corresponding games. The updating of the distributions $f_{1}(k)$ and $f_{4}(k)$ are shown in Fig. 1 . The distribution $f_{1}(k)$ of player 1 is identical along the iterations. Although $Q=2$ in the second iteration for player 1 , only very low values of $k$ are affected. The distributions $f_{4}(k)$ of player 4 show more clearly the update of the belief about the value of $k$.

The last step of the algorithm's iteration is the update of the strategy $x_{i}$ for each player $i \in \mathbb{P}$, according to a best-response dynamic. Let $f_{i}(k)$ denotes the updated i's belief distribution about the $\mathrm{R}$ sensitivity. The estimated value of $k$ by player $i$ corresponds to the expected value $k_{\text {exp }}=\int_{0}^{k_{\max }} k f_{i}(k) d k$. To define the best-response offer $x_{i}$, let us first restrict the set of strategies $S$ to:

$$
S_{i}=\left\{x \in S: x-k_{\exp }\left(N_{i}-x\right)>0\right\},
$$

Table 4 The values of the estimated value of $k$ and the the best-response offer $x_{i}$ at the end of each iteration of Algorithm 1

\begin{tabular}{|c|c|c|c|c|c|c|c|c|c|c|c|}
\hline Iter. & Player & $k_{\exp }$ & $x_{i}$ & Iter. & Player & $k_{\text {exp }}$ & $x_{i}$ & Iter. & Player & $k_{\text {exp }}$ & $\overline{x_{i}}$ \\
\hline \multirow[t]{5}{*}{1} & 1 & 4.15 & 5 & 2 & 1 & 4.15 & 5 & 3 & 1 & 4.15 & 4 \\
\hline & 2 & 3.07 & 5 & & 2 & 3.11 & 4 & & 2 & 3.15 & 4 \\
\hline & 3 & 3.79 & 5 & & 3 & 3.79 & 4 & & 3 & 3.79 & 4 \\
\hline & 4 & 2.88 & 5 & & 4 & 3.00 & 4 & & 4 & 3.11 & 4 \\
\hline & 5 & 4.17 & 6 & & 5 & 4.17 & 5 & & 5 & 4.17 & 4 \\
\hline
\end{tabular}


which are strategies with a nonnegative utility for a fictive $\mathrm{R}$ player. This latter represents the projection of the player $i$ has for responders in $\mathbb{P}$. The the best-response strategy is then:

$$
x_{i}=\arg \max _{x \in S_{i}} U_{P, \text { accept }}^{i}(x),
$$

which is the offer that maximizes $i$ 's utility in the proposer role. Table 4 shows the updates of the values of $k_{\exp }$ and $x_{i}$ for all players in our instance run.

Finally, the termination criterion of Algorithm 1 is given by step 6. When a proportion $0<r \leq 1$ of the population, usually closer to 1 , adopts the same strategy, then a norm has emerged. This strategy is considered to be the social norm of the population, and the last distribution $P$ of strategies is returned by the algorithm.

\section{Proposed relaxations}

An important assumption of Bicchieri's model is that the strategy distribution $P$ of the population is considered to be a common knowledge among individuals. As seen previously, the computation of the norm estimations $N_{i}$ for the players is mainly based on the distribution $P$. In addition to the assumption "common knowledge $P$ ", we consider two relaxed versions, where each player can only access the distribution of strategies over its (first-order) neighbors: "first-order $P$ " assumption, and over its first and secondorder neighbors, which are neighbors of neighbors: "second-order $P$ " assumption. This is a realistic assumption, since individuals have only access to a limited amount of information, usually in their surroundings. The distinction between the interactions and the information neighborhoods for players has been previously considered for local-interaction games by Durieu and Solal [9] and by Alós-Ferrer and Weidenholzer [1]. The former authors confirm a previous result of Ellison [12], but with a higher speed of convergence. Ellison [12] aimed to find conditions for which the players learn to coordinate on the efficient action, conditions that were under the assumption that each player's interaction neighborhood is included in his information neighborhood. Our goal here is empirical. We aim to determine in an empirical fashion the effects a change of the information neighborhood could have on the spread in the emergence of social norms.

By asserting that each player is able to connect to all others, step 3 of Algorithm 1 implicitly considers a complete network structure of the population. This assumption is rather unrealistic for societies, and even for small communities. The novelty of our account is to consider instead network topologies that could arise in real-world situations, namely Barabási-Albert (BA) [3] and Watts-Strogatz (WS) [26] models, plus the Erdős-Rényi (ER) random graph [13] for benchmark purposes. The aim is to examine how the speed of norm emergence, designated by the total number of the algorithm iterations, is framed in terms of some network parameters. The network density and diameter as well as three different centrality measures are used for this purpose. We also make use of the proper parameters of the network models: $p_{E R}$ the probability to connect vertices in ER models, $p_{W S}$ the probability to rewire links in WS models and $m$ the number of links to add in each time step for BA networks. For each case instance of $m, p_{W S}$ and $p_{E R}$ parameters and the complete graph case, 100 runs of Algorithm 1 are performed 

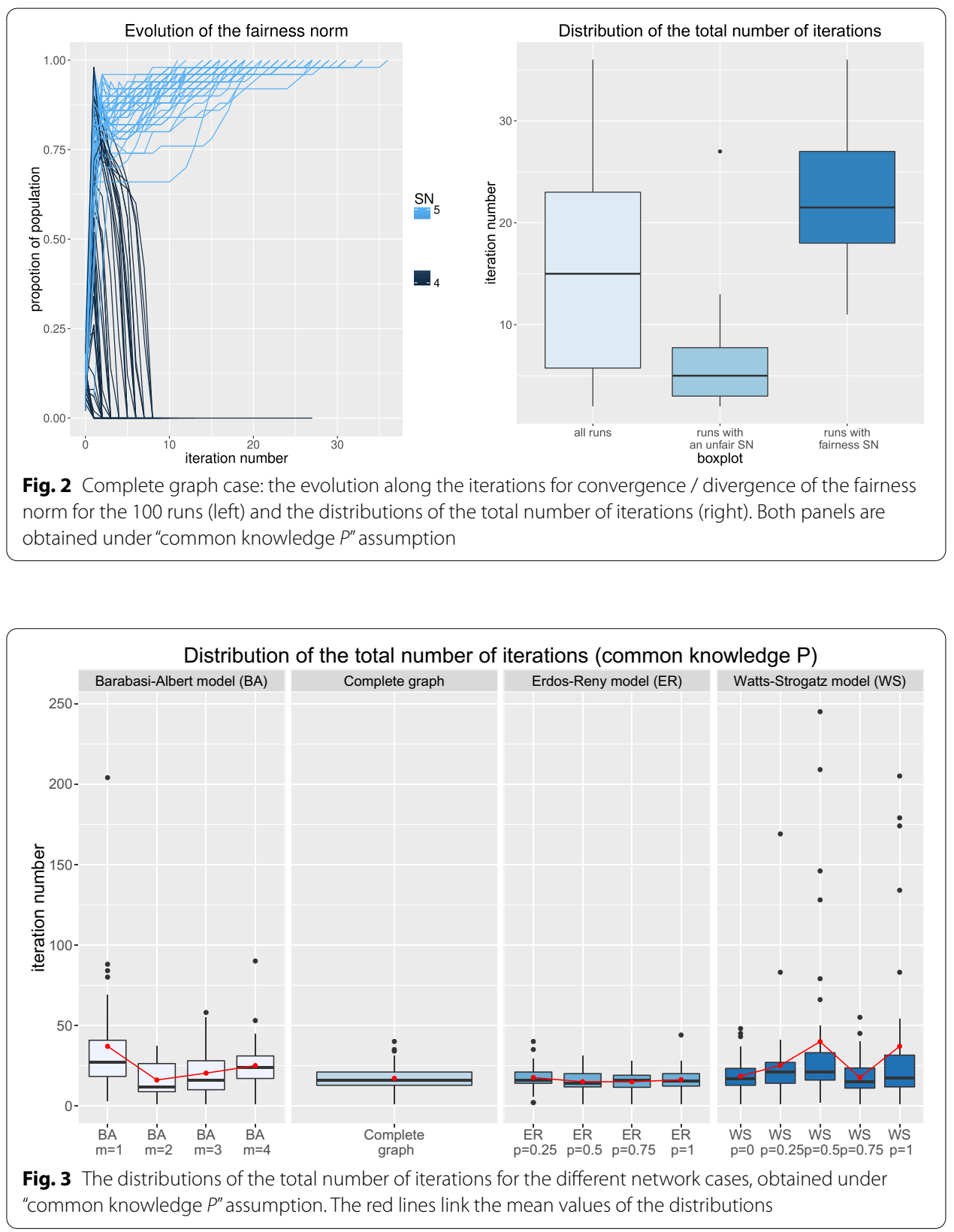

using the $\mathrm{R}$ software. Discussion of the simulation results and their associated figures are given in the next section.

\section{Experimental results}

Algorithm 1 is run with the parameter values employed in Bicchieri [5]: $N=50$, $M=k_{\max }=10, I=5$, and $r=1$. For the complete graph case, we obtain a quick convergence time, on average less than 15 iterations which confirms the results already reported by Bicchieri [5]. However, convergence to the fairness norm is not always guaranteed over the 100 runs, only $54 \%$ of our runs reach the equal split norm, unlike in [5]. As shown in Fig. 2, a non-negligible part of the runs converges to the unfair SN of 


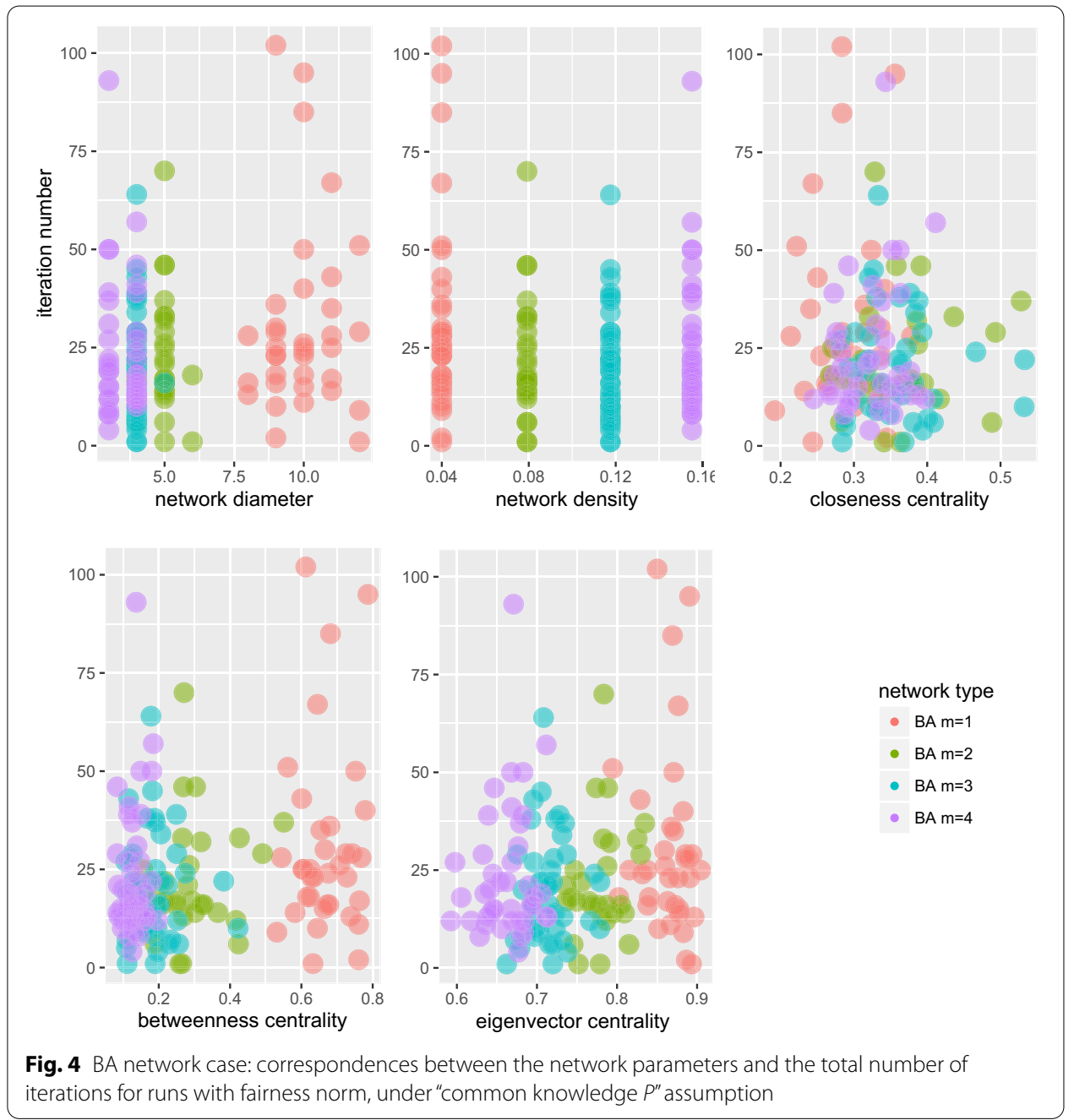

4, which are displayed by the darker curves dropping quickly to zero proportion of the population in the figure's left panel. The right panel of Fig. 2 shows that runs with fairness norm converge with a higher iteration number, around 22 iterations on average.

In the following figures, only runs with the fairness norm are displayed, plus the convergence ratio is lowered to $98 \%$ of the population instead of $100 \%{ }^{3}$. For BA cases, we choose a linear preferential attachment, i.e., its power is fixed to one, and for the original lattice of WS networks only neighbors and next neighbors are connected. Fig. 3 shows that the distribution of the total iteration numbers are quite comparable for the various network topologies. Runs of "BA $m=1$ " have the highest mean of convergence time, which is reasonable since the network structure is actually a tree. In addition, the WS network instances present several outlier runs with slow convergence time.

The network instances are randomly generated. Thus, for each algorithm run, we can associate the corresponding network parameters: diameter, density and centrality index taken at the graph level, to the convergence time, i.e., the total number of iterations.

${ }^{3}$ This setting allows us to considerably reduce the CPU running time. 


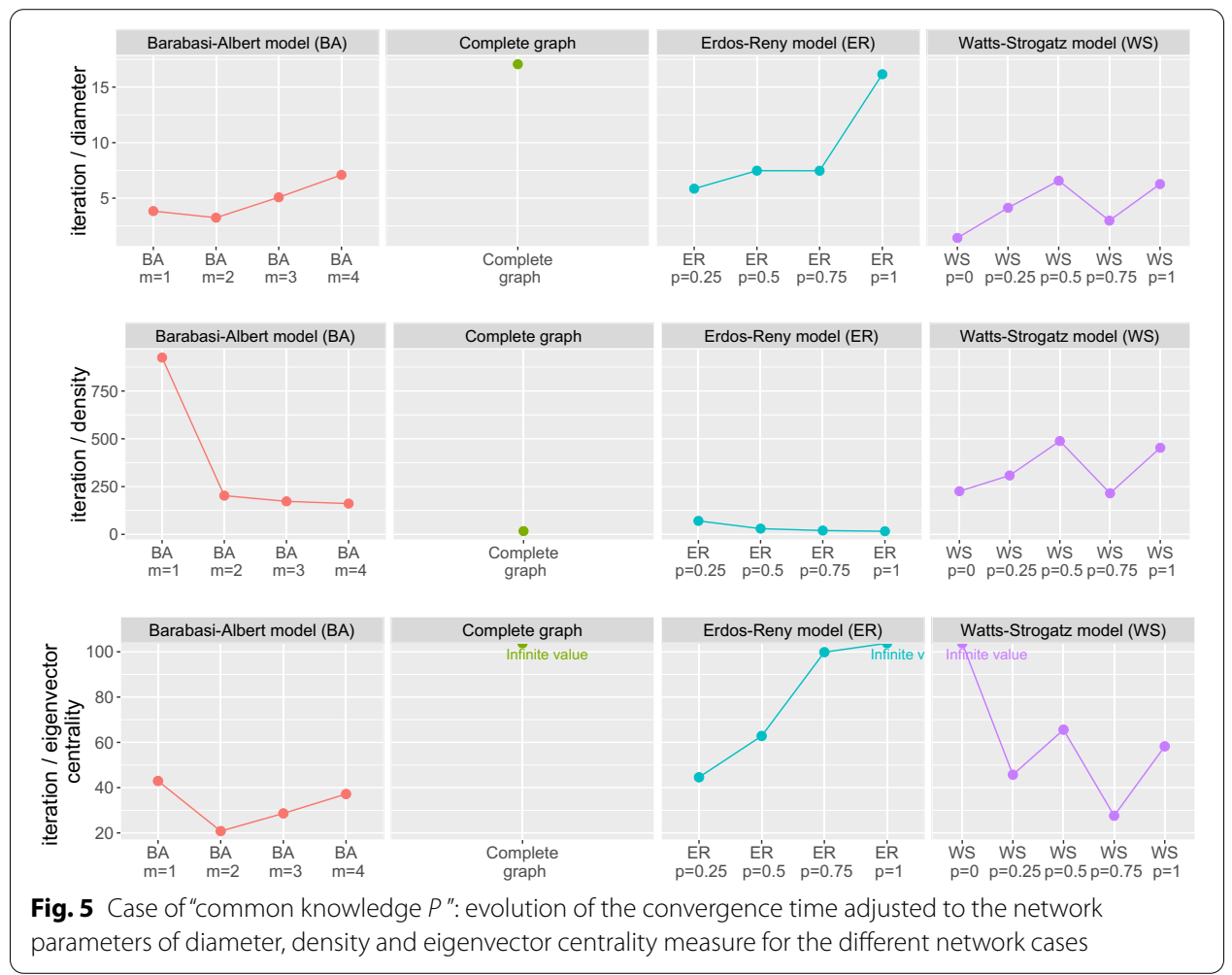

These correspondences are shown in Fig. 4 for BA networks. In this figure for instance, runs of "BA $m=1$ " tree have the largest network diameter and the smallest network density. Using these correspondence, we could easily adjust the convergence time of Fig. 3. In Fig. 5, we examine the adjusted convergence time, wherein the mean of the ratios (iteration/diameter), (iteration/density) and (iteration/centrality index) are independently averaged over the 100 runs and plotted for each network type. The term iteration in the ratios indicates the convergence time. This adjustment can allow comparisons on equal basis and reduce the random effect of network generation.

For BA networks, the ratio (iteration/diameter) is increasing in terms of the parameter $m$, as depicted also in Fig. 3. The trend of Fig. 5 is however more pronounced, mainly due to the 'hubs' characterizing BA networks which reduce the overall distances. A lower network diameter is likely to be obtained as the number of hubs increases with larger $m$. On the other hand, $m$ is inversely correlated with the network density, thus the decreasing tendency of (iteration/density) in BA networks.

For WS model, as $p_{W S}$ probability increases, the original lattice is transformed to a small-world network and finally to a random graph at $p_{W S}=1$. The added shortcuts help to decrease distances. However, the effect of this addition on the plot (iteration/diameter) seems to be rather small. The shape of Fig. 3 is clearly reproduced in Fig. 5. Same remark for the shape of (iteration/density) plot and Fig. 3. We remind that WS small-world networks instances have a constant density.

Concerning ER random graphs, it was proven that their diameter is lower than or equal to 2 for the threshold $p_{E R}>\sqrt{2} \sqrt{\left(\frac{\ln N}{N}\right)} \approx 0.28$ (if $N=50$ ) [7]. This is translated in the evolution of (iteration/diameter), which is rapidly increasing over the threshold 


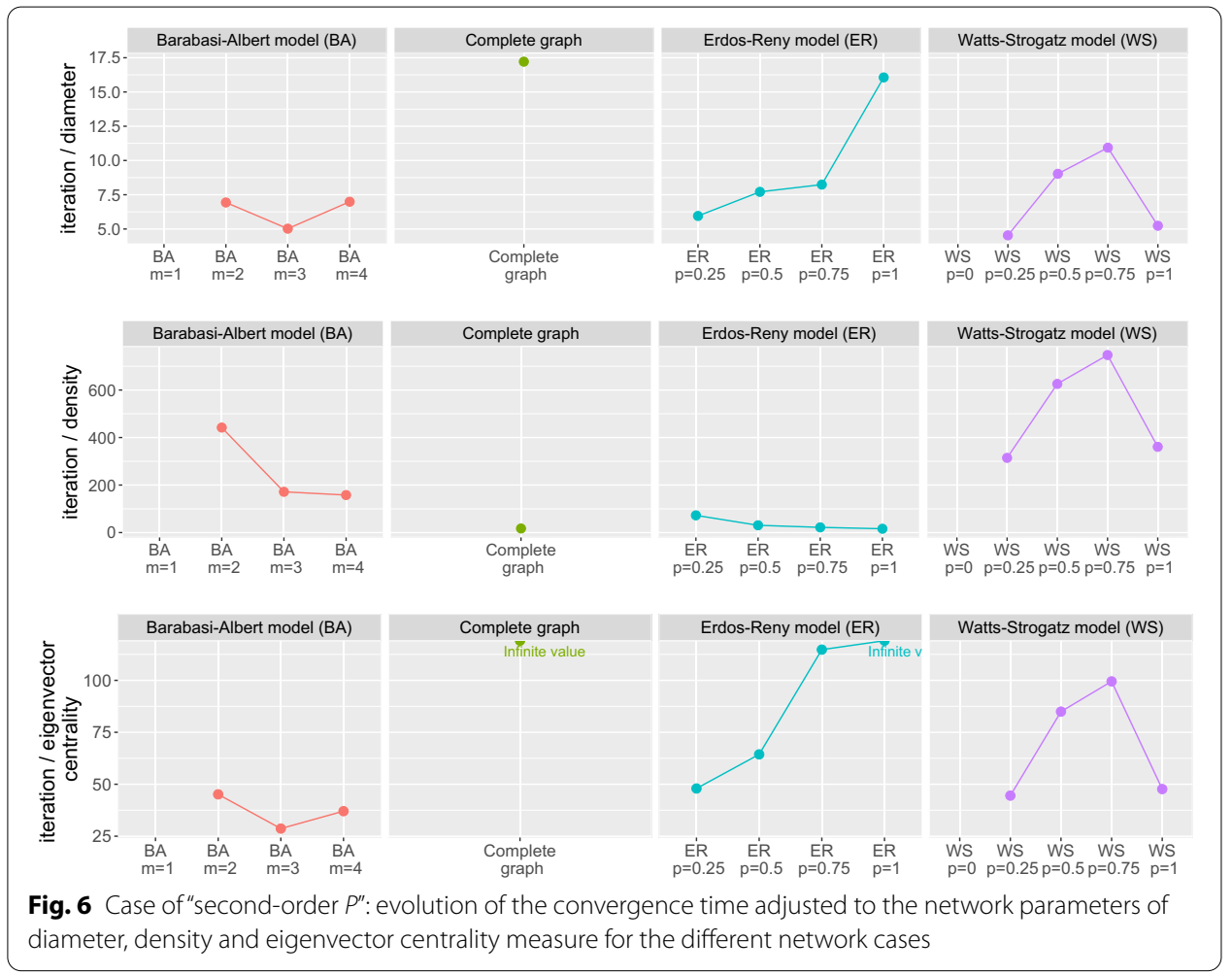

unlike in Fig. 3. We have to say that this evolution is also impacted by a better connectivity obtained with increasing $p_{E R}$ values. Note that the "ER $\mathrm{p}=1$ " instance is a complete graph case.

Centrality index taken at a graph-level is a measure of dispersion. It is higher when larger centrality scores are concentrated on a small number of nodes. Its expression is given as the summation of the differences between the most central node and the centrality index of the other nodes, respectively. For closeness and betweenness centrality, the star network is the most centralized structure $[16,25]$. In the results of Fig. 5, we have obtained that in BA and WS network cases, the shape of eigenvector and closeness (graph-level) centrality plots are quite similar irrespective of the values ranges. This is the case for eigenvector and betweenness centrality plots in ER network. Only plots of eigenvector centrality are shown in Fig. 5. The shapes of the (iteration/eigenvector centrality) plots in BA and WS network cases prove to be generally analogous to those of Fig. 3. Thus, the small effect of the graph-level centrality index compared to the convergence time in these cases. ER random graphs take a different stance with a noticeable increase across $p_{E R}$, which could be somehow understandable since random graphs exhibit generally lower centrality scores due to randomness especially for larger values of $p_{E R}$.

When the "second-order $P$ " assumption is adopted instead of "common knowledge $P$ ", the equivalent of the previously reported Fig. 5 is given by Fig. 6 . The first remark is that the two network cases of "BA $m=1$ " tree and "WS $p_{W S}=0$ " lattice are not convergent, thus the corresponding mean values are not drawn. Secondly, if we compare the mean 


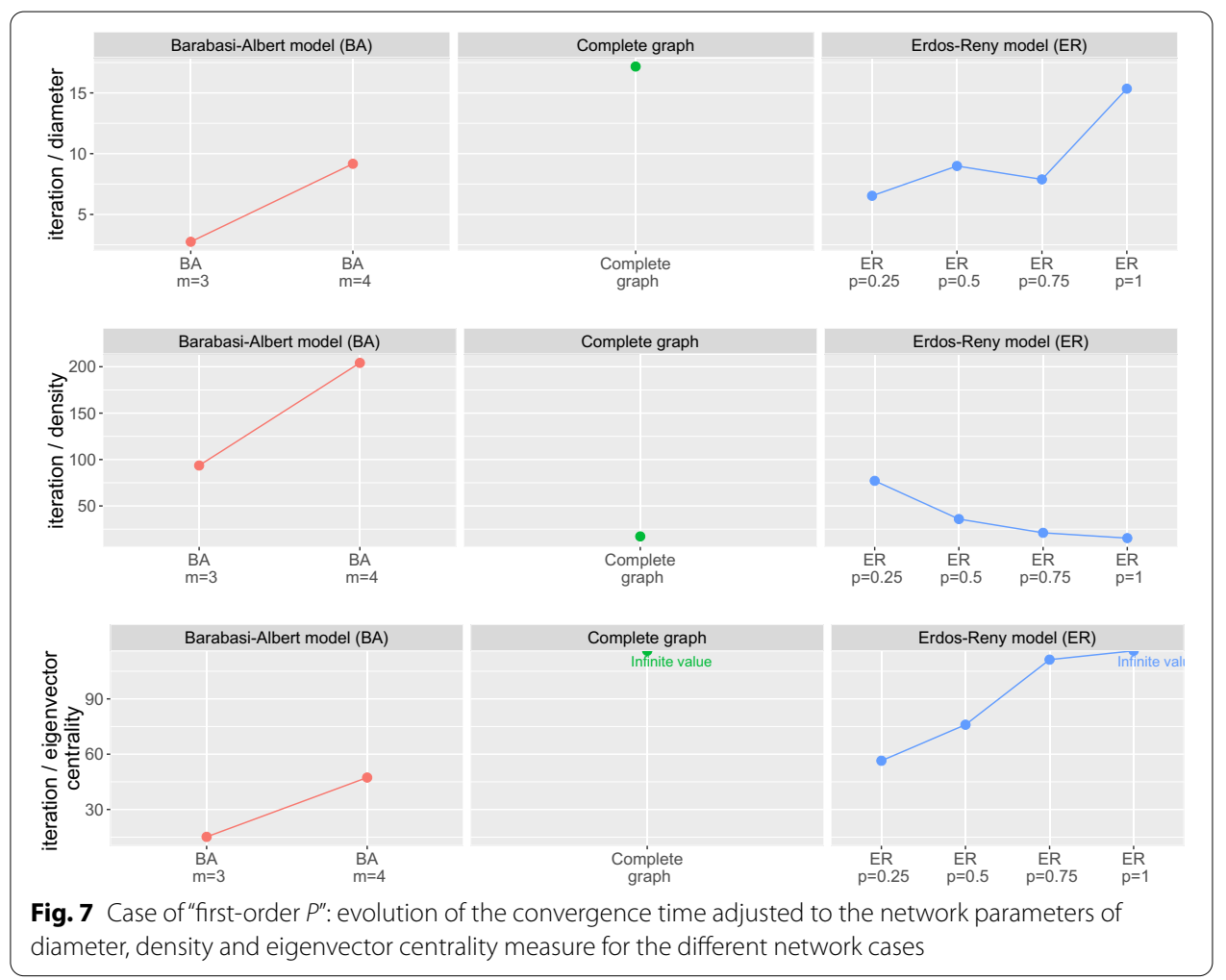

values of the network instances between Figs. 5 and 6, we notice that BA and WS network cases report higher values in Fig. 6, but still within the same ranges of Fig. 5. ER network case shows a particularly close mean values for both assumptions. Additionally, the shapes of the curves of ER networks are also similar for both Figs. 5 and 6 . The shapes of the curves of BA networks reproduce the original evolution in Fig. 3, while WS networks describe a new concave shape. From these comparative observations, we could state that WS network instances are the most affected from the relaxation of the information assumption about the shared offers, and the least affected network cases will be ER random graphs.

Fig. 7 corresponds to the case where the "first-order $P$ " assumption is considered. This case is important as it comforts us in the initial conclusions drawn from Fig. 6. Plots of WS networks are not at all drawn here as none of its network instances is convergent, which means that among the 100 runs no instance run reaches the fairness norm. In BA network case, "BA $m=2$ " is not convergent in addition to the trees "BA $m=1$ ". For ER networks, the mean values reported in Fig. 7 are slightly higher than those of Fig. 6, however all ER network instances converge.

Table 5 shows the convergence time, i.e., the total number of iterations, for the different network cases and information assumptions. To summarize, if $P$ distribution is common knowledge, all network cases converge. For second-order $P$, except the two cases of "BA $m=1$ " tree and "WS $p_{W S}=0$ " lattice, there is convergence. However, for the first-order $P$ assumption, half of the cases do not converge, mainly the WS network instances, and for tree and lattice cases the convergence is not obtained for any social norm so far. ER random graphs seem to be robust to the relaxation of the information 
assumption. Convergence time of ER graphs are not affected by changes of the shared strategies information, according to Table 5 . Investigating the reason behind this seeming robustness of ER random graphs could be an interesting future question to explore. The implementation of the overall simulation approach is made in the $\mathrm{R}$ environment, using 'igraph' [8] and 'doParallel' packages. The various tests are performed on a 2.4-GHz Intel Xeon CPU and 64 GB memory machine.

\section{Conclusion}

This paper is about the emergence of Social Norms (SN) in a social network structure. The study case of fairness norm in the ultimatum game context [5] using the three network structures of Barabási-Albert (BA), Watts-Strogatz (WS) models and ErdősRényi (ER) random graph. The first conclusion is somehow expected. It is that network structure and how information is shared within the network influence in a significant and systematic way the convergence/divergence of the fairness social norm. For instance, "BA $m=1$ " and "WS $p_{W S}=0$ " network cases are not convergent for the fairness norm from the second-order $P$ assumption, wherein the probability distribution of strategies $P$ of a player is shared with its first- and second-order neighbors, while it is steadily converging when $P$ is common knowledge. The second conclusion concerns the convergence feedback along the two relaxations of the information assumption about players' strategies. Empirical results suggest that ER random graphs are the most robust to these relaxations, contrary to WS networks that exhibit a sensitive dependence to the shared $P$ distribution.

Table 5 Average of the convergence time for the fairness SN. 'NA' indicates that fairness norm is not obtained, while ' $N A^{* \prime}$ indicates that all SNs, fairness and unfair are not obtained

\begin{tabular}{|c|c|c|c|c|}
\hline \multicolumn{2}{|c|}{ Information assumption } & \multirow{2}{*}{$\begin{array}{l}\text { Common knowledge } \\
P\end{array}$} & \multirow{2}{*}{$\begin{array}{l}\text { Second-order } P \\
N A\end{array}$} & \multirow{2}{*}{$\begin{array}{l}\text { First-order } P \\
N A^{*}\end{array}$} \\
\hline & $m=1$ & & & \\
\hline & $m=2$ & 16.04 & 35 & NA \\
\hline & $m=3$ & 20.31 & 20.17 & 11 \\
\hline & $m=4$ & 24.98 & 24.5 & 31.67 \\
\hline \multirow{3}{*}{$\begin{array}{l}\text { Complete } \\
\text { WS }\{\end{array}$} & Graph & 17.06 & 17.21 & 17.17 \\
\hline & $p_{W S}=0$ & 18.44 & NA & $N A^{*}$ \\
\hline & $p_{W S}=0.25$ & 25.15 & 25.67 & NA \\
\hline \multirow{7}{*}{ ER \{} & $p_{W S}=0.5$ & 39.87 & 51.08 & NA \\
\hline & $p_{W S}=0.75$ & 17.55 & 61 & NA \\
\hline & $p_{W S}=1$ & 36.97 & 29.45 & NA \\
\hline & $p_{E R}=0.25$ & 17.56 & 17.85 & 19.6 \\
\hline & $p_{E R}=0.5$ & 14.96 & 15.42 & 17.97 \\
\hline & $p_{E R}=0.75$ & 14.93 & 16.48 & 15.76 \\
\hline & $p_{E R}=1$ & 16.16 & 16.06 & 15.35 \\
\hline
\end{tabular}


To modelize SNs, economists and social scientists seem to place more focus on the game-theoretic framework $[5,6,17,22]$, nonetheless the question of "who play the SN game?" is as important as "what SN game is played?". To better account for the 'social' character of social norms, the network structure is a vital and necessary element to incorporate in prospective game-theoretic models.

\section{Acknowledgements}

We would like to thank unknown reviewers for the helpful suggestions to improve this manuscript.

\section{Authors' contributions}

$\mathrm{OR}$ and $\mathrm{HO}$ designed the methodology. OR conducted the empirical experiments. Both authors read and approved the final manuscript.

\section{Funding}

This research received no specific grant from any funding agency in the public, commercial, or not-for-profit sectors.

Availability of data and materials

The datasets used and/or analyzed during the current study are available from the corresponding author on reasonable request.

\section{Declarations}

\section{Competing interests}

The authors declare that they have no competing interests.

\section{Author details}

${ }^{1}$ Departement 14S, Centre CIS, Mines Saint-Etienne, 42023 Saint-Etienne, France. ${ }^{2}$ Department of Mathematical Informatics, Graduate School of Informatics, Nagoya University, Nagoya, Japan.

Received: 16 August 2019 Accepted: 24 September 2021

Published online: 09 October 2021

\section{References}

1. Alós-Ferrer, C., Weidenholzer, S.: Contagion and efficiency. J. Econ. Theory 143(1), 251-274 (2008)

2. Axelrod, R.: An evolutionary approach to norms. Am. Political. Sci. Rev. 80(04), 1095-1111 (1986)

3. Barabási, A.-L., Albert, R.: Emergence of scaling in random networks. Science 286(5439), 509-512 (1999)

4. Bewley, T.F.: Chapter five. Fairness, reciprocity, and wage rigidity. Princeton University Press, Princeton (2012)

5. Bicchieri, C.: The grammar of society: the nature and dynamics of social norms. Cambridge University Press, Cambridge (2005)

6. Binmore, K., et al.: Natural justice. OUP Catalogue (2005)

7. Blum, A., Hopcroft, J., Kannan, R.: Foundations of Data Science. http://www.cs.cornell.edu/jeh/

8. Csardi, G., Nepusz, T.: The igraph software package for complex network research. InterJ. Complex. Syst. 1695(5), 1-9 (2006)

9. Durieu, J., Solal, P.: Adaptive play with spatial sampling. Games. Econ. Behav. 43(2), 189-195 (2003)

10. Durlauf, S.N., Blume, L., et al.: The new Palgrave dictionary of economics, vol. 6. Palgrave Macmillan, Basingstoke (2008)

11. Easley, D., Kleinberg, J.: Networks, crowds, and markets: reasoning about a highly connected world. Cambridge University Press, Cambridge (2010)

12. Ellison, G. Learning, local interaction, and coordination. Econometrica. 1, 1047-1071 (1993)

13. Erdős, P., Rényi, A.: On random graphs. Publicationes Mathematicae Debrecen. 6, 290-297 (1959)

14. Fehr, E., Schmidt, K.M.: A theory of fairness, competition, and cooperation. Q. J. Econ. 114(3), 817-868 (1999)

15. Forsythe, R., Horowitz, J.L., Savin, N.E., Sefton, M.: Fairness in simple bargaining experiments. Games. Econ. Behav. 6(3), 347-369 (1994)

16. Freeman, L.C.: Centrality in social networks conceptual clarification. Soc. Netw. 1(3), 215-239 (1978)

17. Gintis, H.: Social norms as choreography. Politics. Philos. Econ. 9(3), 251-264 (2010)

18. Goyal, S.: Connections: an introduction to the economics of networks. Princeton University Press, Princeton (2012)

19. Güth, W., Schmittberger, R., Schwarze, B.: An experimental analysis of ultimatum bargaining. J. Econ. Behav. Org. 3(4), 367-388 (1982)

20. Lawrence, E.J., Shaw, P., Baker, D., Baron-Cohen, S., David, A.S.: Measuring empathy: reliability and validity of the empathy quotient. Psychol. Med. 34(5), 911-920 (2004)

21. Morris, S.: Contagion. Rev. Econ. Stud. 67(1), 57-78 (2000)

22. Paternotte, C., Grose, J.: Social norms and game theory: harmony or discord? Br. J. Philos. Sci. 64(3), $551-587$ (2013)

23. Schelling, T.C.: The strategy of conflict. Harvard University Press, Cambridge (1980)

24. Sekara, V., Stopczynski, A., Lehmann, S.: Fundamental structures of dynamic social networks. Proc. Natl. Acad. Sci. 113(36), 9977-82 (2016)

25. Wasserman, S., Faust, K.: Social network analysis: methods and applications, vol. 8. Cambridge University Press, Cambridge (1994) 
26. Watts, D.J., Strogatz, S.H.: Collective dynamics of 'small-world' networks. Nature. 393(6684), 440-442 (1998)

27. Weidenholzer, S.: Coordination games and local interactions: a survey of the game theoretic literature. Games. 1(4), $551-585(2010)$

28. Young, H.P.: The economics of convention. J. Econ. Perspect. 10(2), 105-122 (1996)

\section{Publisher's Note}

Springer Nature remains neutral with regard to jurisdictional claims in published maps and institutional affiliations.

Submit your manuscript to a SpringerOpen ${ }^{\circ}$ journal and benefit from:

- Convenient online submission

- Rigorous peer review

- Open access: articles freely available online

- High visibility within the field

- Retaining the copyright to your article

Submit your next manuscript at $\boldsymbol{\Delta}$ springeropen.com 